\title{
Thermal and magnetic measurements on $\mathrm{YBa}_{2} \mathrm{Cu}_{3} \mathrm{O}_{7-y}{ }^{*}$
}

\author{
J E GORDON ${ }^{\dagger}$, R A FISHER, S KIM and N E PHILLIPS \\ Materials and Chemical Sciences Division, Lawrence Berkeley Laboratory, University of \\ California, Berkeley, CA 94720, USA \\ +Permanent Address: Physics Department, Amherst College, Amherst MA 01002, USA.
}

\begin{abstract}
Specific heat measurements in zero and $7 T$ magnetic fields from 0.4 to $100 \mathrm{~K}$, and magnetic susceptibility measurements above $T_{c}$ on a series of polycrystalline samples of $\mathrm{YBa}_{2} \mathrm{Cu}_{3} \mathrm{O}_{7-y}$ (YBCO) reveal a number of regularities. The size of $\Delta C\left(T_{c}\right) / T_{c}$ for the samples $\left[\Delta C\left(T_{r}\right)\right.$ is the jump in the specific heat at $\left.T_{r}\right]$ appears to vary linearly with the low-temperature value of the Debye $\Theta$, with the entropy change between $\approx 85 \mathrm{~K}$ and $T_{c}$ induced by the application of a $7^{5}$ magnetic field, and with the variation of the observed low temperature $y$ with magneuc field, $\mathrm{d} y / \mathrm{d} H$. On the other hand the temperatureindependent part of the magnetic susceptibility above $T_{c}$ appears to be essentially independent of $\Delta C\left(T_{c}\right) / T_{c}$. These results are consistent with the idea that samples of YBCO below $T_{c}$ are a mixture of superconducting and normal phases.
\end{abstract}

Keywords. Specific heat; magnetic susceptibility; $\mathrm{YBa}_{2} \mathrm{Cu}_{3} \mathrm{O}_{7-y}$.

\section{Introduction}

We have made specific heat $C$ and magnetic susceptibility measurements on numerous polycrystalline samples of $\mathrm{YBa}_{2} \mathrm{Cu}_{3} \mathrm{O}_{7-y}(\mathrm{YBCO})$ prepared in a number of different laboratories and by several preparation methods. The principal aim of these measurements has been to determine whether there exist correlations in the height of the specific heat jump at $T_{c}, \Delta C\left(T_{c}\right) / T_{c}$, with other measured quantities. We have previously noted (Phillips et al 1989, hereafter referred to as P1) that the size of $\Delta C\left(T_{c}\right) / T_{c}$ appears to depend on the existence of localized $\mathrm{Cu}^{2+}$ moments that are thought to reside in the YBCO lattice. The results reported in P1 suggest that, because of the pair-breaking role of these moments, a fraction of a typical YBCO sample remains normal below $T_{c}$. They also suggest that a fully superconducting sample would have $\Delta C\left(T_{c}\right) / T_{c} \approx 76 \mathrm{~mJ} / \mathrm{mole} \mathrm{K}^{2}$ and no linear term in the low temperature specific heat. The more typical YBCO sample, in addition to having a term in $C$ that is linear at low temperatures, the size of which is related to $\Delta C\left(T_{c}\right) / T_{c}$, should have other measurable properties which are correlated with the jump in the specific heat at $T_{c}$.

\section{Correlations}

Figure 1 is a graph of $C / T$ vs $T$ for a typical YBCO sample between 80 and $95 \mathrm{~K}$ in zero applied field and in a field of $7 T$. The shaded area in the figure represents

\footnotetext{
${ }^{+}$For correspondence

* Supported by the Director, Office of Energy Research, Office of Basic Energy Sciences, Materials Sciences Division of the U.S. Dept. of Energy under Contract DE-AC03-76SF00098, and by an EXXON Education Grant from the Research Corporation.
} 


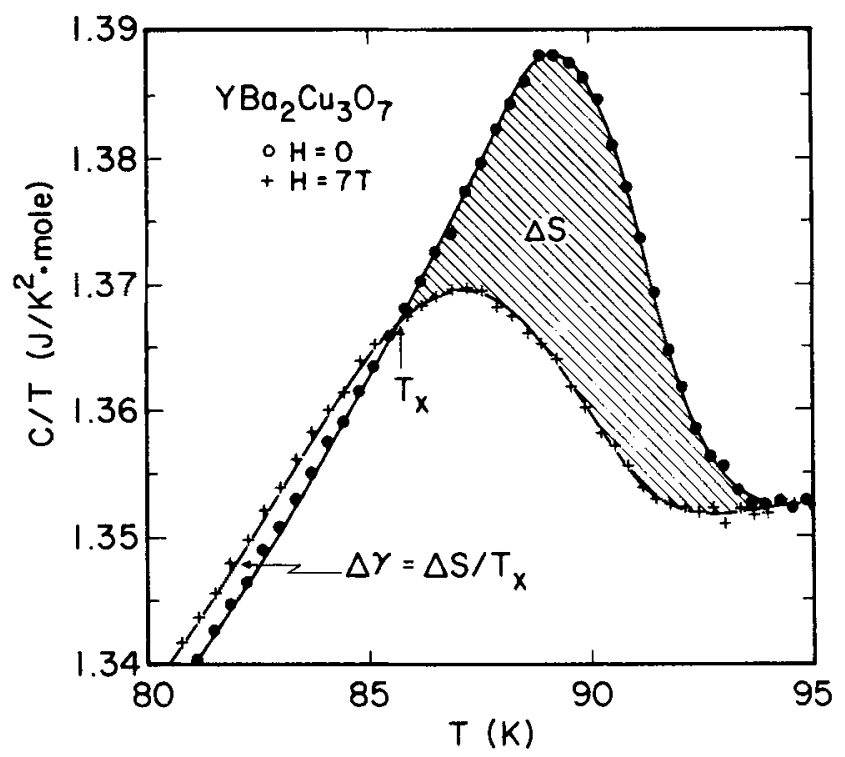

Figure 1. $C / T$ versus $T$. The shaded area represents the entropy difference $\Delta S$, between $T_{x}$ and $T_{\mathrm{c}}$ for the sample in zero and $7 T$ magnetic fields. See text for a discussion of the relation between $\Delta \gamma$ and $\Delta S$.

the entropy decrease $\Delta S$ between $T_{x}\left(\approx 86 \mathrm{~K}\right.$ for this sample) and $T_{c}$ that is produced by the application of the field. If the zero field superconducting transition is incomplete (inclusions of normal material below $T_{c}$ ), then both the specific heat anomaly at $T_{c}$ and $\Delta S$ should reflect this fact. In figure $2 \Delta S$ vs $\Delta C\left(T_{c}\right) / T_{c}$ for various samples is plotted where this latter quantity is determined from an entropy-conserving construction on the zero field $C / T$ vs $T$ data (see Fisher et al 1988). It is evident that an essentially linear relationship between $\Delta S$ and $\Delta C\left(T_{c}\right) / T_{c}$ holds.

Also plotted in figure 2 is $\mathrm{d} \gamma / \mathrm{d} H$, where $\mathrm{d} \gamma / \mathrm{d} H$ is the change with applied magnetic field of the measured low temperature $\gamma$. The increase in $\gamma$ with increasing applied field is consistent with the behaviour observed in type-II superconductors (Radebaugh and Keesom 1966). That $\mathrm{d} \gamma / \mathrm{d} H$ is a linear function of $\Delta C\left(T_{c}\right) / T_{c}$ is understandable if one assumes that both are extensive variables which depend upon the fraction of a sample which is superconducting in zero applied field. It should be noted that $\mathrm{d} \gamma / \mathrm{d} H$ and $\Delta S$ are not independent of one another. The Third Law of Thermodynamics requires that $S\left(T_{c}, 7 T\right)=S\left(T_{c}, 0\right)$. If we assume that at temperatures below $T_{x}$ (see figure 1), $C(T, 7 T)-C(T, 0)=\Delta \gamma T$, where $\Delta \gamma=(\mathrm{d} \gamma / \mathrm{d} H) 7 T$, then the Third Law requires that $\Delta \gamma T_{x}$ should equal the shaded area in figure 1. For all our samples this equality is found to hold within a few per cent.

Figure 2 also indicates that $\chi_{0}$, the temperature-independent part of the magnetic susceptibility above $T_{c}$, is essentially independent of $\Delta C\left(T_{c}\right) / T_{c}$, in contrast to the results obtained in Junod et al (1988). If, with Junod, we assume that the measured $\chi_{0}$ is not very different from $\chi_{p}$, the Pauli susceptibility (the core diamagnetic and the Van Vleck paramagnetic terms are assumed to cancel, approximately), then we can estimate the value of $\gamma$, the Sommerfeld constant for the normal material from $\chi_{0}$. Our value of $2.9 \times 10^{-4} \mathrm{emu} / \mathrm{mole}$ YBCO yields a value for $\gamma$ of $\approx 21 \mathrm{~mJ} / \mathrm{mole}$ 




Figure 2. $\Delta S, \mathrm{~d} \gamma / \mathrm{d} H, \Theta_{0}$, and $\chi_{0}$ versus $T$. See text for the definitions of these quantities.

$\mathbf{K}^{2}$. While it is likely to be fortuitous, this value is in rough agreement with $16 \mathrm{~mJ} / \mathrm{mole}^{2}$, the low temperature $\gamma$ value predicted in $\mathrm{P} 1$ for a sample with $\Delta C\left(T_{c}\right) / T_{c}=0$.

The fourth plot in figure 2 shows that $\Theta_{0}$, the Debye theta obtained from the $T^{3}$ contribution to the low temperature specific heat, also increases with increasing $\Delta C\left(T_{c}\right) / T_{c}$. This result is consistent with that obtained by Eckert (1989), but is difficult to understand in terms of the model proposed in $\mathrm{P} 1$. This variation of $\Theta_{0}$ may indicate either that the localized $\mathrm{Cu}^{2+}$ moments in the YBCO lattice produce a lattice softening or that there is a difference in $\Theta_{0}$ for the superconducting and normal phases. However, so far as we know, neither interpretation has other experimental support.

\section{Conclusions}

As the data plotted in the figures were obtained by looking at small differences between large numbers, there is considerable scatter in some of the plots. In particular some of the parameters for the two $\mathrm{Zn}$-doped samples $\left(\mathrm{Cu}_{2.97} \mathrm{Zn}_{0.03}\right.$ and $\left.\mathrm{Cu}_{2.85} \mathrm{Zn}_{0.15}\right)$ depart considerably from the trends established by data on the other samples, and it may be that for these samples the simple model put forward in P1 must be modified. 
Nonetheless, the above results make it clear that a number of properties of polycrystalline YBCO can be correlated with $\Delta C\left(T_{c}\right) / T_{c}$, and that, therefore, this parameter may well be the best available indicator of sample quality. The model presented in P1 implies that, once impurity-phase effects have been accounted for, other departures from "ideal" superconducting behaviour can be understood by regarding $\mathrm{YBCO}$ as being in a "mixed state" in which the amount of normal material below $T_{c}$ depends upon the concentration of localized $\mathrm{Cu}^{2+}$ moments present in the lattice. Junod et al (1989) showed that a number of properties of YBCO depend upon the oxygen deficiency in the samples. It is possible that this oxygen deficiency is responsible for the localized moments in the lattice, and if so, there may exist a simple relationship between these two quantities that merits investigation.

\section{References}

Eckert D 1989 Chaleur specifiqe a basse temperature des oxyde's supraconducteurs a haute temperature critique, Ph.D. thesis, Universite de Geneve

Fisher R A. Gordon J E and Phillips N E 1988 J. Superconduct. 1231

Junod A, Bezinge A and Muller J 1988 Physica C152 50

Junod A. Eckert D. Graf T, Triscone G and Muller J 1989 Physica C162-164 140 !

Philips N E. Fisher R A. Gordon J E and Kim S 1989 Physica C162-164 1651

Radebaugh R and Keesom P H 1966 Phys. Rev. 149217 